\title{
Preface: A Topical Issue on Solar Image Analysis and Visualization
}

Published online: 22 March 2008

(C) Springer Science+Business Media B.V. 2008

The third Solar Image Processing Workshop (SIPWork III) was held at Trinity College Dublin, Ireland, in September 2006. This meeting brought together researchers in solar physics, image processing, and computer vision, and it focused on preparing for the data analysis and processing needs of new space missions such as the Solar TErrestrial RElations Observatory (STEREO), Hinode, and the Solar Dynamics Observatory (SDO), as well as ground-based instrumentation such as the Advanced Technology Solar Telescope (ATST), the Swedish Solar Telescope (SST), and the Dutch Open Telescope (DOT).

As with SIPWork II (Solar Phys. 228, 2005) we have gathered papers from work presented at SIPWork III as well as related manuscripts in this topical issue of Solar Physics - Solar Image Analysis and Visualization - that the papers mutually benefit from appearing together. The result is 19 papers that apply a range of image processing and computer vision techniques to address the scientific goals of new solar physics instrumentation.

The papers are organized into four groups by types of structures in different levels of the solar atmosphere and visualization tools: $i$ ) software tools for the access and visualization of solar data; ii) granulation and active-region magnetic fields; iii) coronal loops in the solar atmosphere; and $i v$ ) coronal mass ejections.

The organizers thank NASA's Heliophysics Division, the European Office of Aerospace Research and Development (EOARD), and Trinity College Dublin for their support of this meeting. Finally, we thank all those who participated in SIPWork III for making it a very stimulating and successful meeting, and all of the authors and referees who helped make this topical issue possible.

Guest Editors: C. Alex Young and Jack Ireland, ADNET Systems, Inc., Greenbelt, Maryland, USA

Editor: John Leibacher, National Solar Observatory, Tucson, Arizona, USA 